\title{
Comparison of Hippocampal Dendritic Spines in Culture and in Brain
}

\author{
Christopher Boyer, Thomas Schikorski, and Charles F. Stevens \\ Molecular Neurobiology Laboratory, and Howard Hughes Medical Institute at The Salk Institute, La Jolla, California 92037
}

We have quantified hippocampal spine structure at the light and ultrastructural levels in cell cultures $\sim 1-3$ weeks old and in the brains of rodents 5 and $21 \mathrm{~d}$ old. The number of spines bearing synapses increases with age in cultures and in brain, but the structures are similar in both. In culture, about half of the synapses are formed on spines and the remainder are formed on dendritic shafts. In the 5-d-old brain, about half of the synapses occur on dendritic shafts, by 3 weeks of age only $\sim 20 \%$ of synapses are found on dendritic shafts, and in the adult shaft synapses are very rare.

Key words: synapse; culture; ultrastructure; spines; hippocampus; morphometry
In cortex and in the cerebellum, most excitatory contacts are made on spines, those peculiar thorn-like structures believed since Ramón y Cajal $(1891,1896)$ discovered them to be of great, if obscure, functional significance. The structure and ultrastructure of spines have been described in considerable detail for various parts of the brain (neocortex: Ramón y Cajal, 1891, 1896; cerebellum: Harris and Stevens, 1988; hippocampus: Harris and Stevens, 1989; neostriatum: Wilson, 1983; olfactory cortex: Woolf et al., 1991), and various different theories about spine function have been developed (Swindale, 1981; Crick, 1982; Harris and Kator, 1994; Shepherd, 1996). According to one popular view, spines serve as a chemical compartment for second-messenger molecules (Holmes, 1990; Harris et al., 1992; Koch and Zador, 1993), and some experimental evidence has been provided to support this view (Guthrie et al., 1991; Müller and Connor, 1991; Yuste and Denk, 1995; Svoboda et al., 1996). Compartmentalization of second-messenger molecules such as calcium may be important for synaptic plasticity (Lisman, 1989), and changes in spine morphology have also been reported during development (Turner and Greenough, 1985) and with learning (Woolley et al., 1990).

Because synapses are so densely packed in the gray matter of the CNS - on the order of a billion per microliter, studies of synaptic function can sometimes be greatly facilitated by the use of neuronal cell cultures where the synapses are arrayed essentially in two dimensions at relatively low densities. To compare data obtained in culture with observations made in slices and in vivo, however, one must know how culture synapses compare with their brain counterparts. One property of synapses that is important for such comparisons is their structure. Schikorski and Stevens (1997) noted that although the presynaptic structure of culture synapses is quantitatively very similar to the correspond-

Received March 23, 1998; revised May 4, 1998; accepted May 6, 1998.

This work was supported by the Howard Hughes Medical Institute, National Institutes of Health Grant NS 12961 (C.F.S.), and National Institutes of Health, Neuroplasticity of Aging training Grant (T.S.). We thank Dr. Reinhard Jahn for his gift of anti-synaptophysin antibody (G96), Richard Jacobs for his technical assistance, and Carrie Musick for her help with this manuscript.

Correspondence should be addressed to Dr. Charles F. Stevens, Molecular Neurobiology Laboratory, and Howard Hughes Medical Institute at The Salk Institute, 10010 N. Torrey Pines Road, La Jolla, CA 92037.

Copyright (C) 1998 Society for Neuroscience $\quad 0270-6474 / 98 / 185294-07 \$ 05.00 / 0$ ing structural features of synapses in situ, excitatory synapses on dendritic shafts, instead of on spines, are rather common in culture, whereas spine synapses are the rule in brain. This work, however, did not examine postsynaptic structural characteristics in culture.

Papa et al. (1995) provided a quantitative description of spines in culture and documented changes in structure that occur through several weeks of development. At the time their work was performed, however, the dynamic nature of spine-like filopodial protrusions from dendrites was not fully appreciated. Using in vitro staining techniques, recent workers have stressed the marked activity of dendrites that are seen constantly to produce fine filopodia throughout development in culture (Cooper and Smith, 1992; Dailey and Smith, 1996; Ziv and Smith, 1996). These filopodia are similar to spines in structure, appear to be directly involved in synapse formation, and are observed to be spine precursors. This view of the role for filopodia in synaptogenesis originated in the work of Morest (1969a,b). Although Papa et al. (1995) appreciated the difference between filopodia and genuine spines, they did not consistently restrict their analysis to spinelike processes that contacted a presynaptic element. Therefore we have performed a structural analysis similar to the one reported by Papa et al. (1995) but limited to those spine-like elements associated with an immunohistochemically (synaptophysin) defined presynaptic bouton.

Our study had a second motivation. Because spines are believed to be of functional significance, particularly perhaps for synaptic plasticity, it is important to know what fraction of the synapses in culture are made on spines rather than on dendritic shafts. We thus estimated the spine/shaft synapse prevalence for cultures of the type used in physiological investigations and also for the young [postnatal day 21 (P 21)] rat brain, another preparation important for certain physiological studies. If spines are required to provide a separate biochemical compartment for specific synaptic functions, then synapses in culture and those in the young rodent brain may constitute a good model of mature brain synapses only to the extent that culture and young brain synapses occur on spines.

In confirmation of earlier studies, we find that the density of spine synapses increases as cultures mature. At comparable ages, however, spine synapses in culture are more than threefold less 
abundant per dendritic length than they are in brain. Most importantly, perhaps, we find that only $\sim 50 \%$ of the excitatory synapses in culture are on spines, whereas $80 \%$ of such synapses occur on spines in the 2-week-old brain; in the adult brain, excitatory synapses on dendritic shafts are very rare. We believe that these observations have implications for studies of synaptic plasticity (long-term potentiation and depression) in culture and in brain slices from young animals.

\section{MATERIALS AND METHODS}

Primary neuronal cells were grown on inverted glass coverslips suspended above a feeding layer of astrocytes according to the method of Banker and Goslin (1991).

Preparation of the astrocyte feeder layer. To provide support for inverted coverslips, a hot soldering iron was used to make three to four small melted plastic beads in the bottom of each collagen/poly-D-lysine-coated well of a tissue culture dish (Costar, Cambridge, MA). Type 1 astrocytes were purified from 5-d-old heterogeneous hippocampal cell cultures through rigorous agitation for $24-48 \mathrm{hr}$. The purified astrocytes were trypsinized from the bottom of the flask, washed, and replated at a density of 5000 cells per beaded well in $0.5 \mathrm{ml}$ growth medium [DMEM, $10 \%$ heat inactivated fetal calf serum (Life Technologies, Gaithersburg, MD), $20 \mathrm{~mm}$ glucose, $50 \mathrm{U} / \mathrm{ml}$ penicillin, $50 \mathrm{mg} / \mathrm{ml}$ streptomycin, $\mathrm{N}-2$ supplement (1:200, Life Technologies), and phenol red]. Astrocytes were incubated for $3-4 \mathrm{~d}$ at $37^{\circ} \mathrm{C}$ and $5 \% \mathrm{CO}_{2}$ until they developed confluent monolayers. Immediately before neuronal culture preparation, the growth medium was exchanged with neuronal medium [DMEM with 25 mM HEPES, 10\% horse serum (Life Technologies), $20 \mathrm{~mm}$ glucose, penicillin/streptomycin, N-2 supplement (1:100), sodium pyruvate (1 $\mathrm{mM}$ ), and $1 \mathrm{mg} / \mathrm{ml}$ biotin].

Neuronal culture preparation. Hippocampi from 1- to 2-d-old rats (Long-Evans) were dissected in Earl's buffered salt solution (EBSS, Life Technologies) with 10 mM HEPES. The dentate gyrus area was cut off, and the remaining hippocampal tissue was chopped into small blocks. These blocks of tissue were incubated with periodic agitation in Papain $(\sim 20 \mathrm{U} / \mathrm{ml}$ EBSS $)$ for $0.75-1.5 \mathrm{hr}$ at $37^{\circ} \mathrm{C}$. Tissue was washed with growth medium and dissociated into single-cell suspension with multiple passages through a $5 \mathrm{ml}$ plastic pipette. Cells were plated on collagen/polyD-lysine-coated coverslips at a density of 6000-8000 cells per well in 0.5 $\mathrm{ml}$ neuronal medium. After 3-4 hr, coverslips were inverted and placed into the beaded wells containing established astrocyte feeder layers and $0.5 \mathrm{ml}$ neuronal medium. Arabosylcytosine $(5 \mathrm{~mm})$ was added after $1-2$ $\mathrm{d}$ to inhibit proliferation of non-neuronal cells.

Lucifer yellow injections. At different ages (6, 10, 14, $21 \mathrm{~d}$ in vitro), individual coverslips were fixed in $4 \%$ paraformaldehyde for 5-10 min at room temperature. Single cells were impaled under visual control with sharp microelectrodes, and a constant current of $-2-5 \mathrm{nA}$ injected Lucifer yellow for 5-15 min. Successful injected cells were post-fixed further with $4 \%$ paraformaldehyde for $5 \mathrm{~min}$.

Immunocytochemistry. Coverslips with Lucifer yellow-filled cells were permeabilized in methanol for $5-10 \mathrm{~min}$ at $-20^{\circ} \mathrm{C}$, washed, and incubated in a $10 \%$ bovine serum albumin (BSA) in PBS for $20 \mathrm{~min}$. The incubation with the anti-synaptophysin antibody [G96, gift of R. Jahn, Max Planck Institut (diluted 1:2000 in PBS, 1\% BSA)] was performed at $4^{\circ} \mathrm{C}$ overnight, followed by an incubation with an anti-rabbit antibody biotin conjugated (1:300; Vector Laboratories, Burlingame, CA) for $2 \mathrm{hr}$ at room temperature. For detection of biotin the coverslips were incubated with avidin-Texas Red conjugate (1:300, Vector) for $2 \mathrm{hr}$ at room temperature. Coverslips were washed thoroughly and mounted on microscope slides with antifading medium.

Image analysis. Images of all dendrites of successfully filled cells were acquired with a video microscope at a magnification of $60 \times$, with a filter set for Lucifer yellow and for Texas Red. Image pairs were contrastenhanced, and measurements were made by using the MetaMorph Tools (Universal Imaging Corporation, West Chester, PA).

Electron microscopy. One 5-d-old and one 21-d-old Long-Evans rat were perfused through the heart under deep Nembutal anesthesia ( 80 $\mathrm{gm} / 25 \mathrm{gm}$ body weight). A short perfusion with oxygenated saline was followed by a perfusion with $70 \mathrm{ml}$ of $4 \%$ glutaraldehyde in $100 \mathrm{~mm}$ phosphate buffer, $\mathrm{pH}$ 7.4. The brains were dissected and immersed in the same fixative overnight at room temperature. Vibratome sections including the hippocampus were cut and post-fixed in $1 \% \mathrm{OsO}_{4}$ for $1 \mathrm{hr}$ at $4{ }^{\circ} \mathrm{C}$.
After dehydration in ethanol, sections were contrasted en bloc in $0.5 \%$ uranyl acetate and flat-embedded in Epon.

Cultured hippocampal cells were fixed and processed as described previously (Schikorski and Stevens, 1997). Serial sections were cut from both specimens and analyzed (for details, see Schikorski and Stevens, 1997).

\section{RESULTS}

Quantitative analysis of serial electron micrograph sections is the preferred way to study a structure, such as a spine, that is so close to the light microscope limit of resolution. Unfortunately, such an analysis is prohibitively difficult for a large sample of synapses. Therefore, we have made the same compromise advocated by earlier investigators: use of the light microscope to obtain a relatively large sample size ( $\sim 7000$ spines here) supplemented with limited electron microscopic analysis for confirmation of major conclusions based on the lower resolution observations. We have filled cells with Lucifer yellow to visualize spines and have identified synapses with synaptophysin immunohistochemistry (Fig. 1).

Because most electrophysiological studies of cultured neurons use cells grown for 2-3 weeks in vitro, we have studied cultures that were maintained for $6,10,14$, and $21 \mathrm{~d}$. We defined a spine as a fine protrusion from the dendritic shaft that occurs in close apposition to an anti-synaptophysin positive bouton (Fig. 1). A presynaptic element adjacent to the spine head was not the only criterion for inclusion. Fine protrusions with a bouton at their base were counted as well as spines, because we frequently found synapses in culture on spine necks at the ultrastructural level. We identified a synapse as occurring on a dendritic shaft when we could not detect a fine protrusion adjacent to the immunolabeled bouton. Because the dendritic shaft in some cases could obscure a short spine, our inclusion criteria probably led to some undercounting of spine synapses. We return to this issue in Discussion. Electron microscopy was performed on cultures at $14 \mathrm{~d}$ in vitro (DIV; approximately equivalent to postnatal age) and for comparison on a rat brain at P5 and P21 (Fig. 2).

\section{Spine densities}

We found that spine densities are low at 6-10 DIV and then double or triple in number by 14-21 DIV (Fig. 3). Specifically, the younger cultures exhibit approximately 0.2 spine synapses per micrometer of dendritic length, whereas the older cultures (2-3 weeks) have approximately 0.5 spine synapses per micrometer of dendritic length. Compare these spine densities with modern estimates of 1.7 spine synapses per micrometer of dendritic length in adult hippocampus (Trommald et al., 1995) This lower density in culture, together with the sparseness of dendrites and their restriction to two dimensions, is of course what makes culture systems appealing for synaptic physiologists.

Interestingly, the density of synapses (the number of spine synapses per length of dendrite) changes little from 2 to 3 weeks in culture. At 2 weeks, we counted 0.44 spines per micrometer of dendritic length, and at 3 weeks the density was 0.45 spines per micrometer. One would expect synaptogenesis to continue in the third week, so this constancy presumably represents a dynamic balance between synapse formation and elimination.

In addition, we measured all spine-like objects (innervated and not innervated) on dendrites at the various times in vitro. This measure should be comparable to the data of Papa et al. (1995). The densities of spine-like protrusions were $0.39 / \mu \mathrm{m}$ after $6 \mathrm{DIV}$, $0.33 / \mu \mathrm{m}$ after $10 \mathrm{DIV}, 0.59 / \mu \mathrm{m}$ after $14 \mathrm{DIV}$, and $0.62 / \mu \mathrm{m}$ after 21 DIV. By calculating the difference between true spines and 
Figure 1. Original fluorescence staining of the same dendrite of a hippocampal cell in culture. $A$, Dendrite after filling with Lucifer yellow. $B$, The corresponding immunocytochemical staining against synaptophysin. The arrows in both images point to the sites of spines, and the arrowheads point to the site shaft synapses.
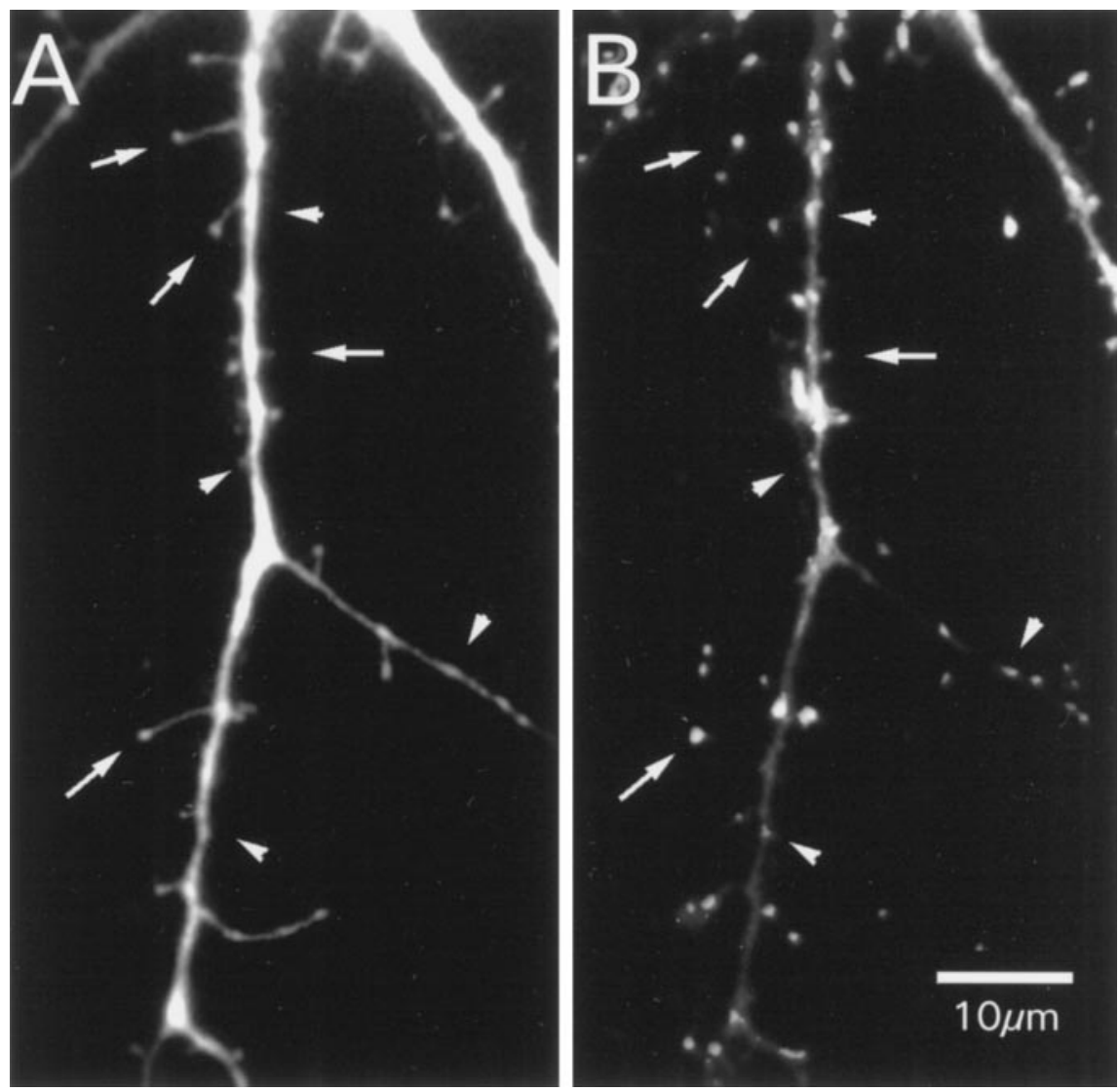

noninnervated filopodia, we found a constant value of $\sim 0.2$ filopodia per micrometer at all ages. This constant value presumably indicates that dendrites are seeking new synapses at a constant rate per dendritic length throughout development in culture.

\section{Shaft versus spine synapses}

In brain, excitatory synapses on the shafts of spiny dendrites are very rare (Harris and Kater, 1994). In our cultures, however, about half of the synapses were on the dendritic shaft (Fig. 3). Somewhat surprisingly, this is true at every age in culture. Thus, physiological studies using cultures draw on a population of synapses that may differ, on average, from those in brain with respect to their postsynaptic structural properties.

\section{Length distributions}

The distributions of spine lengths for each of the age groups are presented in Figure 4. In our images a single pixel had the length of $0.17 \mu \mathrm{m}$, and we have sorted spine lengths into bins of $0.5 \mu \mathrm{m}$, which corresponds to approximately three image pixels. Any spines shorter than $\sim 0.5 \mu \mathrm{m}$ were not included in our measurements. As is apparent from all of the distributions, a spine length of $1-1.5 \mu \mathrm{m}$ is most common for every age. In the 2- to 3 -week-old cultures, however, the prevalence of longer spines decreases, so that the mean spine length decreases with age (Fig. 4, inset). By the time cultures are 2-3 weeks old, almost all of the spines are 1-1.5 $\mu \mathrm{m}$ long.

\section{Electron microscopic studies of synapses in culture}

Because structural features of spines are near or below the resolution of the light microscope, we felt that it was important to confirm our major light microscopic observations with electron microscopy, especially the ratio of spine/shaft synapses. Therefore we have counted shaft and spine synapses in culture in electron microscopic images from serial sections and have confirmed that they appear in approximately equal numbers. In a sample of 29 synapses, we found 14 on spines and 15 on the dendritic shaft at 14 DIV in culture. All of these synapses were morphologically classed excitatory, with the typical asymmetric structure and round synaptic vesicles. We found only two symmetric contacts on dendritic shafts, and these were not included within our sample. The spine head volume was on average $0.058 \pm 0.034 \mu \mathrm{m}^{3}$. For data on postsynaptic density area and number of docked vesicles, see Schikorski and Stevens (1997).

\section{Ultrastructure of synapses at P5}

At P5, for 14 CA1 synapses in two series of sections (27 sections total), we found $5(36 \%)$ spine synapses and $9(64 \%)$ shaft synapses. All spines were innervated by only one presynaptic bouton, and no bouton formed more than one synapse. The synaptic density is very low compared with that in older animals (in our series, approximately six times lower than at P21), which is the reason for our small sample size. For a quantification of synaptic structure at this age, we pooled all synapses; the result is shown in Table 1. With the exception of bouton volume, all structural features of synapses are highly correlated, whereas the bouton volume is correlated significantly only with the total number of vesicles. Because we encountered only five spines in our sample, we did not include spine head volume data in the table. The spine head volumes were $0.0323,0.0516,0.0716$, 0.0837 , and $0.1314 \mu \mathrm{m}^{3}$. 

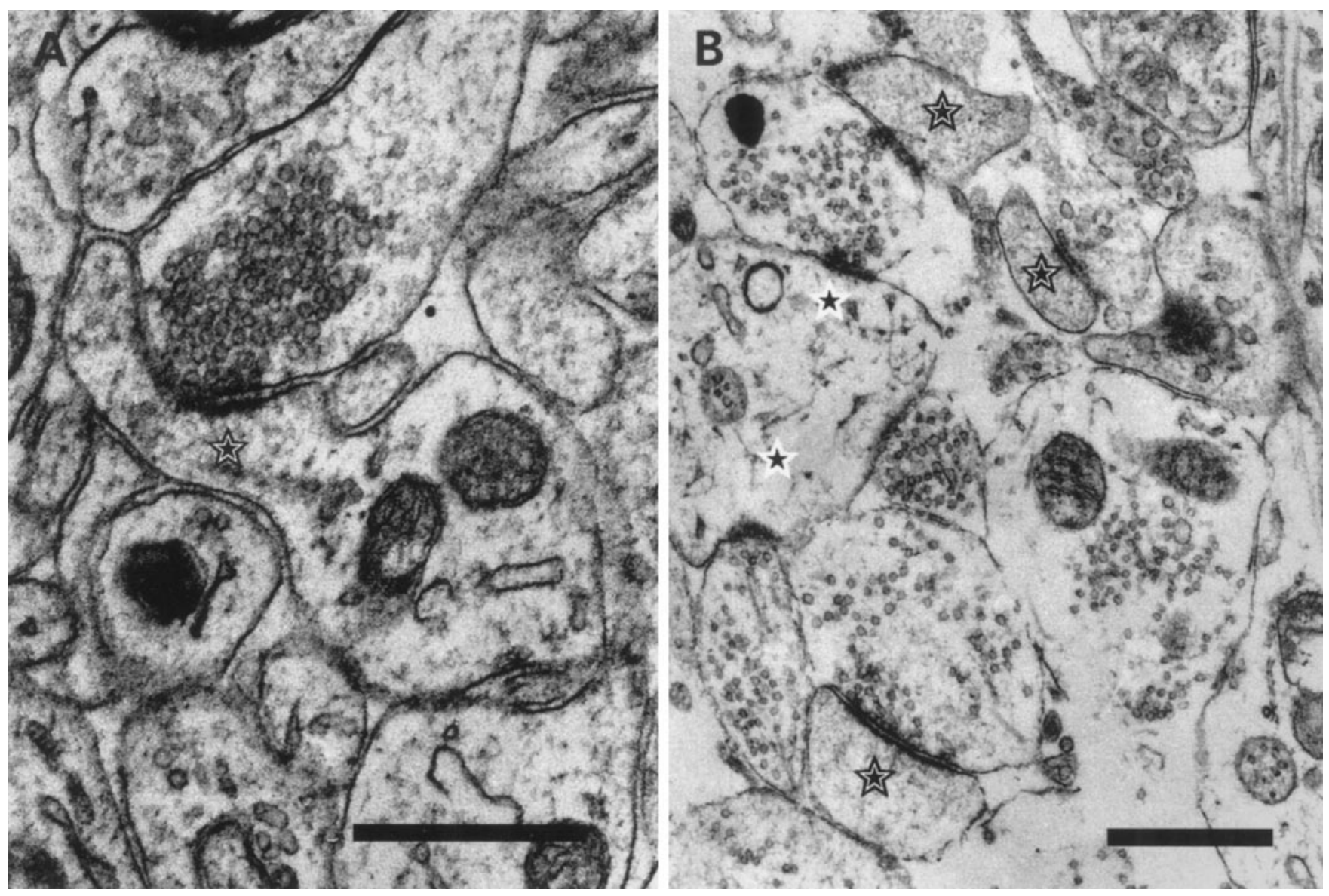

Figure 2. Ultrastructure of developing synapses. A, The largest spine (star) we encountered in our sample of synapses at P21. All typical structural features are present at that age; the postsynaptic density is aligned with the active zone. The spine had the typical grainy dense appearance, and the spine apparatus is visible. $B$, Spines from hippocampal neurons in culture after 14 DIV. Spines (stars) in culture show no qualitative difference from their counterparts in situ. Shaft synapses are marked with a star on a white background. Scale bars, $0.5 \mu \mathrm{m}$.

\section{Ultrastructure of synapses at P21}

Because many physiological experiments use hippocampal slices from several week-old rodents, we have, in addition to examining spines in culture, quantified some of the aspects of spine morphology at P21 at the ultrastructural level. It is potentially important to know how different the spine morphology is compared with the adult animal for the interpretation of physiological experiments. We found that $82 \%(71 / 87)$ of the synapses in a region of stratum radiatum were found on spines; thus, spine synapses predominate at $\mathrm{P} 21$ in contrast to the $\mathrm{P} 5$ hippocampus, where most synapses are on dendritic shafts. Nevertheless, nearly one fifth (16/87) of the excitatory synapses in hippocampus (CA1) at P21 were formed on shafts. We encountered in the young animals the entire spectrum of spine morphologies that have been described for the adult. From the total number of 87 synapses, we reconstructed 26 synapses fully contained in serial sections. two spines $(7.7 \%)$ made contact with two different boutons, and one spine $(3.8 \%)$ was innervated by three boutons. All other spines formed only one synapse. On the presynaptic side, one (3.8\%) bouton formed four synapses (which had four distinct active zones opposite four different postsynaptic elements), and five boutons formed two synapses. Two of those boutons formed one spine and one shaft synapse. Schikorski and Stevens (1997) described various presynaptic morphological features quantitatively and found that they are highly correlated. We have extended this

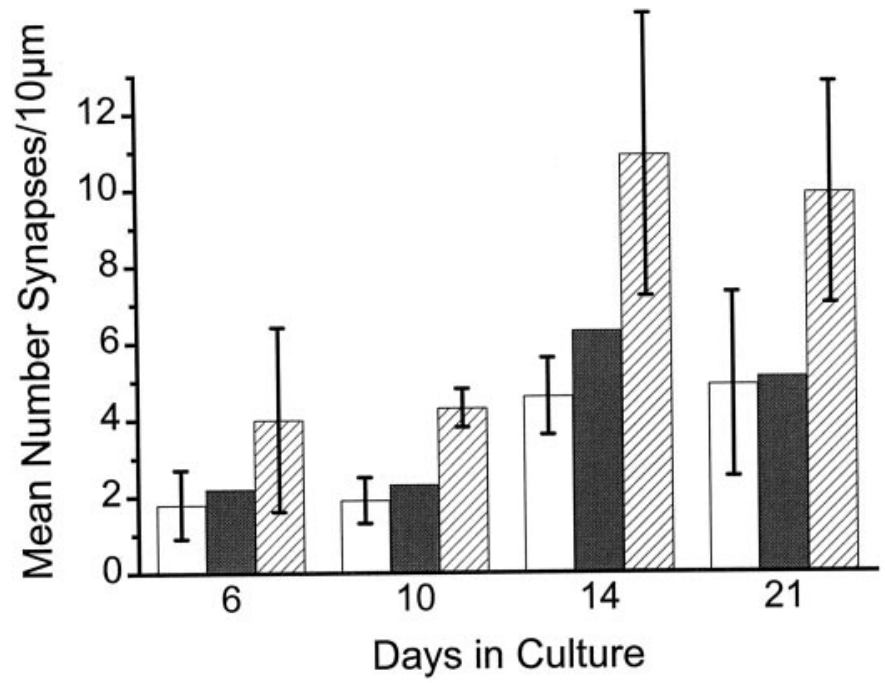

Figure 3. Comparison of synaptic density in culture as a function of time. The different bars depict the number of spine synapses (white bars), the number of shaft synapses ( gray bar, calculated as the differences from total synapses minus spine synapses), and the number of all synapses (striped bars). Between 10 and 14 DIV there is a significant increase of synaptic density. Before and after this age the density of spine and shaft synapses is constant. Note that the relationship between shaft and spine synapses is equal for all ages. 
Figure 4. Spine length distribution in culture at different ages. Most of the spines have a length of $1-1.5 \mu \mathrm{m}$. Note that the occurrence of longer spines in young cultures is significantly increased. The inset depicts the decreasing mean spine length and SE over time.

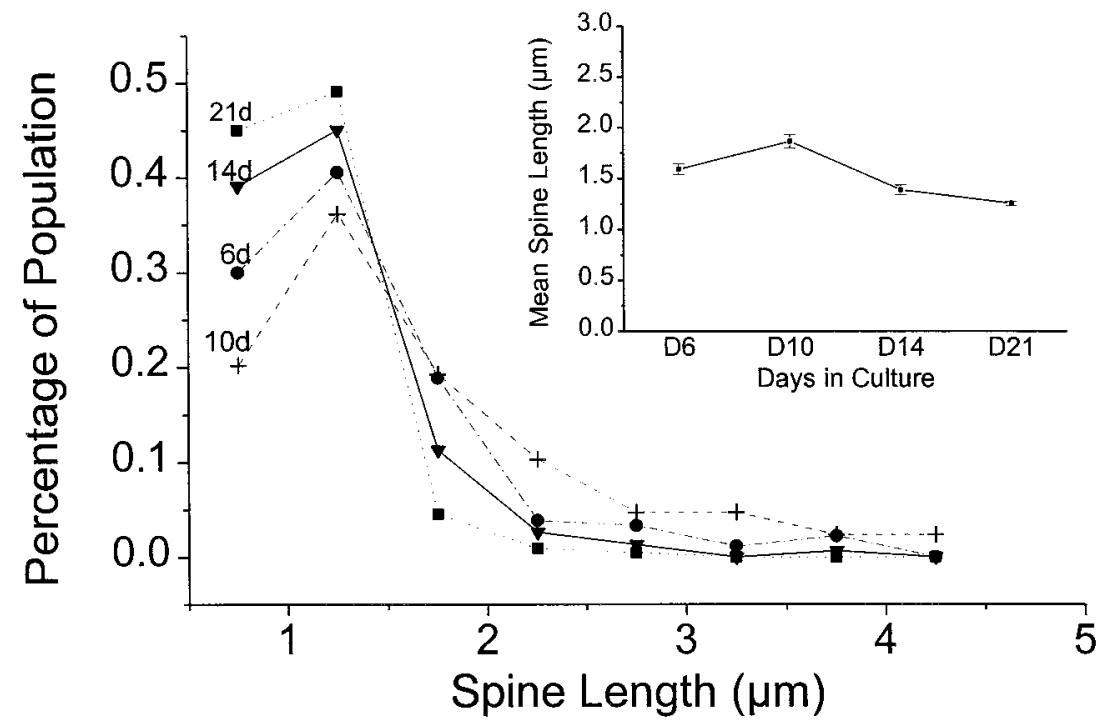

Table 1. Sizes of various structures at hippocampal synapses at P5 and their intercorrelations

\begin{tabular}{lccccccc} 
& Mean & SD & Range & AZ & PSD & DV & TV \\
\hline AZ $\left(\mu^{2}\right)$ & 0.08 & 0.035 & $0.034-0.142$ & 1 & 0.97 & 0.80 & 0.49 \\
PSD $\left(\mu^{2}\right)$ & 0.073 & 0.037 & $0.037-0.147$ & & 1 & 0.83 & 0.46 \\
DV & 13.6 & 7.7 & $5-31$ & & & 0.29 \\
TV & 162 & 102 & 0.0415 & $0.059-0.189$ & & 0.28 \\
BV $\left(\mu^{3}\right)$ & 0.1195 & 0.320 & & 0.22 \\
\end{tabular}

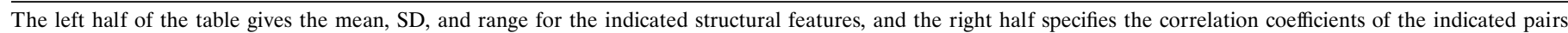


0.0837 , and $0.1314 \mu \mathrm{m}^{3}$. AZ, Active zone; PSD, postsynaptic density; DV, docked vesicle number; TV, total vesicle number; BV, bouton volume; SV, spine volume.

Table 2. Sizes of various structures at hippocampal synapses at P21 and their intercorrelations

\begin{tabular}{lccllllll} 
& Mean & SD & Range & AZ & PSD & DV & TV & BV \\
\hline AZ $\left(\mu^{2}\right)$ & 0.03 & 0.014 & $0.0087-0.0566$ & 1 & 0.77 & 0.9 & 0.28 & 0.16 \\
PSD $\left(\mu^{2}\right)$ & 0.029 & 0.013 & $0.0087-0.0666$ & & 1 & 0.65 & 0.31 & 0.1 \\
DV & 8.8 & 4.4 & $3-20$ & & & 1 & 0.44 \\
TV & 160 & 134 & $15-571$ & & & 0.62 \\
BV $\left(\mu^{3}\right)$ & 0.084 & 0.052 & $0.0121-0.1833$ & & & 0.6 \\
SV $\left(\mu^{3}\right)$ & 0.022 & 0.014 & $0.0031-0.0572$ & & & & 0.4 \\
\end{tabular}

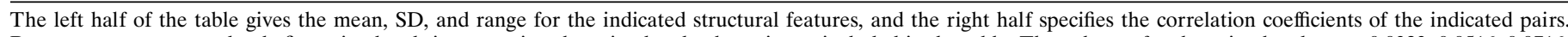


0.0837, and $0.1314 \mu \mathrm{m}^{3}$. AZ, Active zone; PSD, postsynaptic density; DV, docked vesicle number; TV, total vesicle number; BV, bouton volume; SV, spine volume.

quantitative analysis to include some postsynaptic features in the young rodent brain. Most of the structured characteristics of the synapses are correlated with each other (Table 2). For example, active zone area, postsynaptic density area, spine head volume, total number of vesicles, and number of docked vesicles are all highly correlated. The one exception is the bouton volume, which does not show correlations with other structures in the young hippocampus. Because of the difficulty in estimating the length of spine necks that run tangentially through many serial sections, we did not quantify lengths for the spines reconstructed at the ultrastructural level.

\section{DISCUSSION}

We found that dendrites of hippocampal cells in culture possess at most only one third of the spine density observed on their counterparts in brain at a comparable age. Furthermore, about half of the excitatory synapses in culture were on the dendritic shaft, whereas the vast majority of excitatory synapses in mature brain are formed on spines (Harris and Kater, 1994). In the P5 hippocampus, shaft synapses are more common than those on spines, whereas spine synapses predominate by 3 weeks of age; nevertheless, we found $\sim 20 \%$ of the synapses on the dendritic shaft in the 3-week-old hippocampus.

Our data confirm and extend those published previously (Papa et al., 1995). We found spine densities at the various times in vitro that were similar to those reported earlier, although we limited our sample to spine-like processes with a immunohistochemically defined presynaptic element. The agreement of our data with those of Papa et al. (1995), who counted all spine-like processes, results from the presence of a constant number of filopodia that are noninnervated at all ages. Also the spine length distribution 
shows the same trend. Our observation of an approximately constant spine/shaft synapse ratio in culture is interesting and unexpected because it has been hypothesized that shaft synapses mature into spine synapses; therefore, one might expect that shaft synapses would decrease in abundance over time, but they do not. This constant ratio might result from a continuing process of synapse formation, maturation, and elimination in culture.

We are confident that shaft and spine synapses occur at the ultrastructural level in approximately equal numbers as we report here, because we have found this to be true in many electron micrographs of cultures not formally included in the present study. Nevertheless, we must stress the limitations of the use of electron microscopy for the quantitative analysis of synapses in culture. The basic problem is the low density of synapses in cultures (of the type used for electrophysiological studies) as compared with brain. Because synapses are so abundant in brain, a typical set of 30 serial sections contains an adequate sample of synapses for reconstruction and measurement. The same series of sections made from culture will provide only 10-20 synapses. Furthermore, measurement of the length of extended structures, such as spines, is correspondingly more difficult in culture. Because of our small sample of culture synapses, one must recognize that measured sizes are less certain than for brain.

At P5, the ultrastructures of spines and synapses appear quite similar to their counterparts in older animals (Harris et al., 1989; this paper). Quantitatively, however, some important differences can be recognized. Very young brain synapses (P5) share three features with synapses in culture. First, the synaptic density is approximately six times lower than at P21 or in the adult animal. Data from neocortex (Valverde, 1971; Markus and Petit, 1987) also show low synaptic density at this age; thus synaptogenesis in hippocampus seems to have a time course similar to that in neocortex. Second, at P5, 64\% of all synapses are made on dendritic shafts. This is again similar to distribution of synapses in culture. Third, the spines we found are larger than those in older animals but are comparable to the spine sizes in culture. The postsynaptic density/active zone size, however, is larger at P5 than these specializations at synapses in culture.

When we compared our ultrastructural data at P21 with data from Harris et al. (1992), we found similar dimensions. This earlier work, however, did not provide average values for a random set of spines. Our data suggest that we encountered many "thin" spines because our average is closest to the average of thin spines reported by Harris et al. (1992) in young animals. The largest spine that we encountered, however, measured $0.057 \mu \mathrm{m}^{3}$; this is approximately six times smaller than mushroom spines reported by Harris et al. (1992). The recent data from dentate gyrus on spine sizes and shape (Trommald and Hulleberg, 1997) are similar to our new data.

A quantitative comparison of spine synapses at P21 with their counterparts in culture at 14 DIV shows that the postsynaptic density/active zone sizes are comparable, whereas the spine head volume is smaller in brain synapses. The synaptic density at P21 is rather similar to that of the adult and thus much higher then after 14 DIV.

We used $t$ test to compare our data of P21 with the data from adult mouse (Schikorski and Stevens, 1997); we found no significant differences. However, the bouton volume was highly correlated with other structures such as active zone area in the adult animal, whereas boutons in the brain at P21 did not show such a correlation.

Several groups have reported that long-term depression (LTD) in culture is resistant to the NMDA receptor antagonist 5-aminopyridine (AP-5) (Deisseroth et al., 1996; Goda and Stevens, 1996). Spines are frequently considered to be important for forming a compartment that limits calcium ions to the immediate postsynaptic region and also isolates the spine compartment from the dendritic shaft. The increase in calcium concentration necessary to induce LTD could be derived for shaft synapses from dendritic calcium channels rather than from NMDA receptor channels if the spine neck is required to exclude dendritic calcium from the spine head. Our morphological observations are in agreement with this notion because approximately half of the LTD in the Goda and Stevens (1996) experiments, performed in cultures similar to the ones studied here, were resistant to NMDA receptor antagonists, just as approximately half of the synapses were on spines and half were on the dendritic shaft. Presumably, calcium entry through dendritic calcium channels would have ready access to the postsynaptic membrane and could substitute for calcium that normally would have had to enter through NMDA receptor channels. Our observation of a relatively common occurrence of shaft synapses in the CA1 region of young animals (16/87) might explain why LTD in these animals is relatively resistant to AP-5. Oliet et al. (1997) argued for the existence of two distinct forms of LTD, but their data could have been accounted for equally well by a single LTD mechanism expressed at two distinct types of synapses, spine and shaft, with different sources of calcium and different accessibility by drugs.

Long-term potentiation (LTP) is know to "wash out" with whole-cell recording, a phenomenon attributed to the need for a distinct postsynaptic compartment. The greater difficulty in inducing LTP in culture and in slices from young animals might relate to the relatively large fraction of shaft rather than spine synapses in these neurons. In any case, the investigator of synaptic plasticity must be aware that the anatomical situation in culture and young animals is not identical to that in older animals and be alert to possible differences that derive from the structural factors noted here.

The picture of central synaptogenesis originally proposed by Morest (1969a,b) and elegantly confirmed by more recent work (Cooper and Smith, 1992; Saito et al., 1992; Dailey and Smith, 1996; Ziv and Smith, 1996) holds that dendrites effectively increase their target area for axons by extending filopodia that actively search out the exploring axonal growth cones. According to this view, once an axon has made appropriate contact with a filopodium, the filopodium is converted into a synaptic spine. Our observations relative to this process are indirect but nevertheless confirmatory. Presumably spine morphology optimizes some structural feature that is required functionally. Because spine necks are mostly 1-1.5 $\mu \mathrm{m}$ in length (Trommald and Hulleberg, 1997; our data), we may suppose that for some reason this length is optimal or limited. Because filopodia search a space larger than this, the Morest-Smith view of synaptogenesis implies that axon paths should be slightly rearranged to provide the optimal spine length. Our observation that the longer spine necks are eliminated with increasing age fits with this notion.

\section{Conclusion}

Because we do not fully understand the function of spines, the full significance of our findings for understanding synaptic function is still uncertain. Qualitatively, most structural properties of synapses are indistinguishable between culture and hippocampus at P5 forward, but some quantitative features are quite different. The relative abundance of shaft synapses in culture may be the 
most prominent difference. Additionally, spines in culture have a relatively larger volume, comparable to spines at P5. On the other hand, the postsynaptic density/active zone sizes in cultured spines are similar to their in situ counterparts at P21 or in adults. It seems that the reduction in spine size seen in brain may not be necessary in culture, because cells and their processes are not as densely packed as in the brain (a similar statement can be made about the bouton volume in culture and in young animals). The synaptic physiologist must ensure that such differences do not limit the range of validity for conclusions about synaptic function based on observations in culture and in slices from young brains.

\section{REFERENCES}

Banker G, Goslin K (1991) Culturing nerve cells. Cambridge, MA: MIT.

Cooper MW, Smith SJ (1992) A real-time analysis of growth conetarget cell interactions during the formation of stable contacts between hippocampal neurons in culture. J Neurobiol 23:814-828.

Crick F (1982) Do dendritic spines twitch? Trends Neurosci 5:44-46.

Dailey ME, Smith SJ (1996) The dynamics of dendritic structure in developing hippocampal slices. J Neurosci 16:2983-2994.

Deisseroth K, Bito H, Tsien RW (1996) Signaling from synapse to nucleus: postsynaptic CREB phosphorylation during multiple forms of hippocampal synaptic plasticity. Neuron 16:89-101.

Goda Y, Stevens CF (1996) Long-term depression properties in a simple system. Neuron 16:103-111.

Guthrie PB, Segal M, Kater SB (1991) Independent regulation of calcium revealed by imaging dendritic spines. Nature 354:76-80.

Harris KM, Kater SB (1994) Dendritic spines: cellular specializations imparting both stability and flexibility to synaptic function. Annu Rev Neurosci 17:341-371.

Harris KM, Stevens JK (1988) Dendritic spines of rat cerebellar Purkinje cells: serial electron microscopy with reference to their biophysical characteristics. J Neurosci 8:4455-4469.

Harris KM, Stevens JK (1989) Dendritic spines of CA1 pyramidal cells in the rat hippocampus: serial electron microscopy with reference to their biophysical characteristics. J Neurosci 9:2982-2997.

Harris KM, Jensen FE, Tsao BH (1992) Three-dimensional structure of dendritic spines and synapses in rat hippocampus (CA1) at postnatal day 15 and adult ages: implications for the maturation of synaptic physiology and long-term potentiation. J Neurosci 12:2685-2705.

Holmes WR (1990) Is the function of dendritic spines to concentrate calcium? Brain Res 519:338-342.

Koch C, Zador A (1993) The function of dendritic spines. J Neurosci 13:413-422.

Lisman J (1989) A mechanism for the Hebb and the anti-Hebb processes underlying learning and memory. Proc Natl Acad Sci USA 86:9574-9578.

Markus EJ, Petit TL (1987) Neocortical synaptogenesis, aging, and behavior: lifespan development in the motor-sensory system of the rat. Exp Neurol 96:262-278.

Morest DK (1969a) The differentiation of cerebral dendrites: a study of the postmigratory neuroblast in the medical nucleus of the trapezoid body. Z Anat Entwicklungsgesch 128:271-289.

Morest DK (1969b) The growth of dendrites in the mammalian brain. Z Anat Entwicklungsgesch 128:290-317.

Müller W, Connor JA (1991) Dendritic spines as individual neuronal compartments for synaptic Ca2+ responses. Nature 354:73-76.

Oliet SHR, Malenka RC, Nicoll RA (1997) Two distinct forms of longterm depression coexist in CA1 hippocampal pyramidal cells. Neuron 18:969-982.

Papa M, Bundman MC, Greenberger V, Segal M (1995) Morphological analysis of dendritic spine development in primary cultures of hippocampal neurons. J Neurosci 15:1-11.

Ramón y Cajal S (1891) On the structure of the cerebral cortex in certain mammals. La Cellule 7:125-176. In: Cajal on the cerebral cortex (de Felipe J, Jones EG, eds) (1988), pp 23-54. Oxford: Oxford UP.

Ramón y Cajal S (1896) The collateral spines of the cerebrum stained with methylene blue. Revista Trimestral Micrografica, I:123-136, In: Cajal on the cerebral cortex (de Felipe J, Jones EG, eds), pp 94-103 (1988). Oxford: Oxford UP.

Saito Y, Murakami F, Song W-J, Okawa K, Shimono K, Katsumaru H (1992) Developing corticorubral axons of the cat form synapses on filopodial dendritic protrusions. Neurosci Lett 147:81-84.

Schikorski T, Stevens CF (1997) Quantitative ultrastructural analysis of hippocampal excitatory synapses. J Neurosci 17:5858-5867.

Shepard GM (1996) The dendritic spine: a multifunctional integrative unit. J Neurophysiol 75:2197-2210.

Svoboda K, Tank DW, Denk W (1996) Direct measurement of coupling between dendritic spines and shafts. Science 272:716-719.

Swindale NV (1981) Dendritic spines only connect. Trends Neurosci 4:240-241.

Trommald M, Hulleberg G (1997) Dimensions and density of dendritic spines from rat dentate granule cells based on reconstructions from serial electron micrographs. J Comp Neurol 377:15-28.

Trommald M, Jensen V, Andersen P (1995) Analysis of dendritic spines in rat CA1 pyramidal cells intracellularly filled with a fluorescent dye. J Comp Neurol 353:260-274.

Turner AM, Greenough WT (1985) Differential rearing effects on rat visual cortex synapses. I. Synaptic and neuronal density and synapses per neuron. Brain Res 329:195-203.

Valverde F (1971) Rate and extent of recovery from dark rearing in the visual cortex of the mouse. Brain Res 33:1-11.

Wilson CJ, Groves PM, Kitai ST, Linder JC (1983) Three-dimensional structure of dendritic spines in the rat neostriatum. J Neurosci 3:383-398.

Woolf T, Sheperd G, Greer C (1991) Local information processing in dendritic trees: subsets of spines in granule cells of the mammalian olfactory bulb. J Neurosci 11:1837-1854.

Woolley CS, Gould E, Frankfurt M, McEwen BS (1990) Naturally occurring fluctuation in dendritic spine density on adult hippocampal pyramidal neurons. J Neurosci 10:4035-4039.

Yuste R, Denk W (1995) Dendritic spines as basic functional units of neuronal integration. Nature 375:682-684.

Ziv NE, Smith SJ (1996) Evidence for a role of dendritic filopodia in synaptogenesis and spine formation. Neuron 17:91-102. 